\title{
Locally causal dynamical triangulations in two dimensions
}

\author{
R. Loll ${ }^{1,2, *}$ and B. Ruijl ${ }^{3,4, \dagger}$ \\ ${ }^{1}$ Institute for Mathematics, Astrophysics and Particle Physics, Radboud University, Heyendaalseweg 135, \\ 6525 AJ Nijmegen, Gelderland, Netherlands \\ ${ }^{2}$ Perimeter Institute for Theoretical Physics, 31 Caroline Street North, \\ Waterloo, Ontario N2L 2Y5, Canada \\ ${ }^{3}$ Leiden Institute of Advanced Computer Science, Leiden University, \\ Niels Bohrweg 1, 2333 CA Leiden, Zuid-Holland, Netherlands \\ ${ }^{4}$ Theory Group, NIKHEF, Science Park 105, 1098 XG Amsterdam, Noord-Holland, Netherlands
}

(Received 21 July 2015; published 1 October 2015)

\begin{abstract}
We analyze the universal properties of a new two-dimensional quantum gravity model defined in terms of locally causal dynamical triangulations. Measuring the Hausdorff and spectral dimensions of the dynamical geometrical ensemble, we find numerical evidence that the continuum limit of the model lies in a new universality class of two-dimensional quantum gravity theories, inequivalent to both Euclidean and causal dynamical triangulations. The presence of significant finite-size effects, which are present even at the largest volumes investigated, prevents us from reaching a more definite conclusion.
\end{abstract}

DOI: $10.1103 /$ PhysRevD.92.084002

PACS numbers: 04.60.-m, 04.60.Nc, 05.10.Ln

\section{CAUSAL QUANTUM GEOMETRY IN TWO DIMENSIONS}

The approach of causal dynamical triangulations (CDT) [1] provides concrete evidence that one must include causal, Lorentzian properties in the nonperturbative gravitational path integral in order for the associated quantum gravity theory to possess a classical limit. This should be contrasted with purely Euclidean constructions where the path integral is taken over geometric configurations which represent four-dimensional "spacetimes," but do not contain any information about time, light cones or causality. The problem with the latter appears to be that-quite independent of the elementary "building blocks" used for constructing individual Euclidean configurations-their nonperturbative sum is completely dominated by highly degenerate objects whose superposition never leads to a four-dimensional extended universe on macroscopic scales, no matter how one looks at it.

The Euclidean precursor of CDT, so-called dynamical triangulations (DT), is a case in point. In both DT and CDT quantum gravity one looks for scaling limits of regularized path integral expressions, where curved geometries are represented by triangulated, piecewise flat manifolds. However, the infinite-volume limit of the Euclidean theory is dominated by degenerate phases with no obvious physical interpretation in terms of general relativity [2], and phase transitions are of first order $[3,4]$. By contrast, one of the phases of CDT quantum gravity is characterized by a quantum geometry which on large scales exhibits properties of a four-dimensional de Sitter space [5], and the phase space

r.loll@science.ru.nl; rloll@perimeterinstitute.ca

b.j.g.ruijl@liacs.leidenuniv.nl; benrl@nikhef.nl of CDT contains a whole line of second-order critical points [6], which are being investigated as natural candidates for defining the searched-for continuum theory [7].

At the inception of the CDT approach, the toy model version of the theory in two spacetime dimensions played an important role: the nonperturbative CDT path integral over geometries could in this case be evaluated analytically [8], and the resulting two-dimensional quantum gravity theory could be compared with the much-studied theory of two-dimensional Euclidean quantum gravity, which likewise was formulated and solved exactly in terms of dynamical triangulations (see [9] for reviews). The two theories turn out to be inequivalent, and are characterized by different critical exponents (see [10] for a comparison). In terms of continuum formulations, they lie in the universality class of Liouville quantum gravity [11] for DT and two-dimensional, projectable Hořava-Lifshitz gravity [12] for CDT. These models provide the first completely explicit example that "signature matters" in the context of the nonperturbative gravitational path integral, also known as the "sum over histories." As mentioned above, we now have good evidence that the same is true for the physically relevant case of quantum gravity in four dimensions.

This paper expands on the theme of two-dimensional quantum gravity as an interesting testing ground for quantum gravity proper, where both analytical and numerical solution methods can be employed and compared. We study the two-dimensional implementation of a recently introduced version of CDT, which goes by the name of locally causal dynamical triangulations (LCDT) [13] or "CDT without preferred foliation" [14]. The path integral in these CDT models is performed over a class of piecewise flat Lorentzian geometries that is enlarged compared to standard CDT quantum gravity. The geometries are still 
causal, in the sense of having a well-defined light cone structure everywhere, but are not required to have the preferred (discrete) proper-time slicing characteristic of standard CDT configurations. An in-depth numerical investigation of locally causal DT in three spacetime dimensions found nontrivial evidence that key results of CDT quantum gravity, including the volume distribution of three-dimensional de Sitter space, are reproduced in this generalized causal theory [14].

This is an important and concrete piece of evidence that for a judicious choice of the bare coupling constants of the theory, LCDT and CDT quantum gravity lead to equivalent continuum theories. We investigate whether the same is true in two spacetime dimensions. Although this toy model is arguably even less representative of full gravity than the three-dimensional model, the properties of "quantum geometry" are much simpler to analyze in dimension two and may give us a hint of why the two causal theories are equivalent or not, as the case may be.

Since in terms of its configuration space the locally causal model lies in between DT and CDT quantum gravity, solving it will give us a better understanding of the universality classes of theories of quantum geometry in two dimensions. The CDT universality class has so far proven to be quite robust: inclusion of a higher-curvature term [15], a decoration by arches along spatial links (tantamount to including a restricted class of "baby universes") [16,17], an explicit inclusion of a finite number of baby universes within a string field-theoretic setting based on CDT [18] (see also [19]), or starting from a conceptually rather different Hamiltonian "string bit model" [20] all lead to the same scaling limit. In the absence, to date, of an analytic solution of locally causal DT in two dimensions, we present below the results of a numerical investigation. We have examined several observables and measured two critical exponents, the expectation values of the Hausdorff and the spectral dimension of quantum spacetime, to try to understand whether they coincide with those of DT or CDT quantum gravity, or perhaps signal yet another, new universality class of two-dimensional quantum geometry.

We begin our analysis by introducing the locally causal DT model and its geometry in Secs. II-III, and outline the setup for the Monte Carlo simulations in Sec. IV. Closed timelike curves and their role in LCDT are described in Sec. V. Section VI deals with observables, including the volume profile, a characterization of the quantum geometry in terms of minimal loops, and our results for the Hausdorff and spectral dimensions. Our conclusions are presented in Sec. VII. The appendix contains some details on the geometric shape of typical LCDT configurations.

\section{LOCALLY CAUSAL DYNAMICAL TRIANGULATIONS}

We first derive an expression for the gravitational action in locally causal DT in two dimensions. Our starting point is the two-dimensional Einstein-Hilbert action in the continuum,

$$
S=\kappa \int d^{2} x \sqrt{|g|}(R-2 \Lambda),
$$

where $\kappa$ is the inverse of Newton's constant, $\Lambda$ the cosmological constant, and $g$ denotes the determinant of the Lorentzian spacetime metric $g_{\mu \nu}$. The integral over the scalar curvature $R$ is topological and will not play a role in the path integral construction, since the spacetime topology will be held fixed. Dropping the $R$-term leaves us with just the volume term. Absorbing the (dimensionless) gravitational constant into the cosmological constant $\Lambda$, the path integral and its regularized counterpart in terms of dynamical triangulations read

$$
\int \mathcal{D}[g] \mathrm{e}^{-i \Lambda \int d^{2} x \sqrt{|g|}} \rightarrow \sum_{T} \frac{1}{C(T)} \mathrm{e}^{-i \lambda V_{2}(T)},
$$

where the (formal) integration over diffeomorphism equivalence classes $[g]$ of metrics on the left-hand side has been replaced by a sum over inequivalent triangulations $T$ with the usual DT measure involving the order $C(T)$ of the automorphism group of $T$. The constant $\lambda$ on the right-hand side is the bare cosmological coupling of the regularized theory, and $V_{2}(T)$ denotes the spacetime volume of the triangulation $T$.

As described in [14], LCDT in $1+1$ dimensions uses two types of triangular Minkowskian building blocks to allow for the construction of geometries without a preferred time foliation (see Fig. 1). The usual CDT simplex $\Delta_{\text {stt }}$ with one spacelike and two timelike edges is supplemented by another two-simplex $\Delta_{s s t}$ with one timelike and two spacelike edges. The squared edge length of all spacelike links is $l_{s}^{2}=a^{2}$ and that of timelike links $l_{t}^{2}=-\alpha a^{2}$, in terms of the lattice cutoff $a$ and the ratio $\alpha>0$ of the two quantities. To determine the spacetime volume $V_{2}(T)$ of a triangulation $T$ assembled from these building blocks, we simply need to count their numbers $N_{s t t}(T)$ and $N_{s s t}(T)$ and compute the volumes of both $\Delta_{s t t}$ and $\Delta_{s s t}$. The latter are determined in a straightforward way from the values of the edge lengths of the two triangles, and are given by

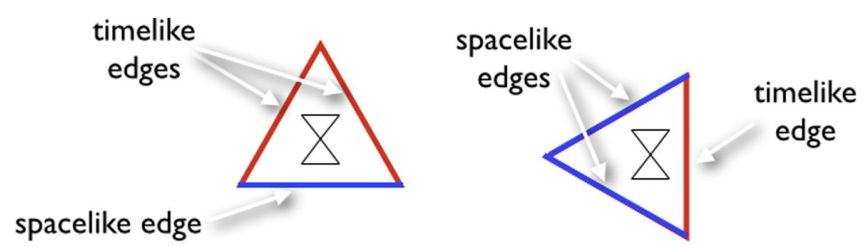

FIG. 1 (color online). The two elementary building blocks of $(1+1)$-dimensional LCDT, with light cones indicated: stttriangle $\Delta_{s t t}$ with one space- and two timelike edges (left) and $s s t$-triangle $\Delta_{s s t}$ with one time- and two spacelike edges (right). The color coding for spacelike edges is blue, and for timelike ones red. 

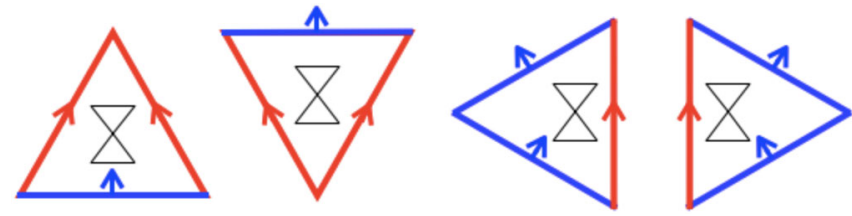

FIG. 2 (color online). Two time orientations are possible for each triangle type, as indicated by the future-pointing arrow assignments.

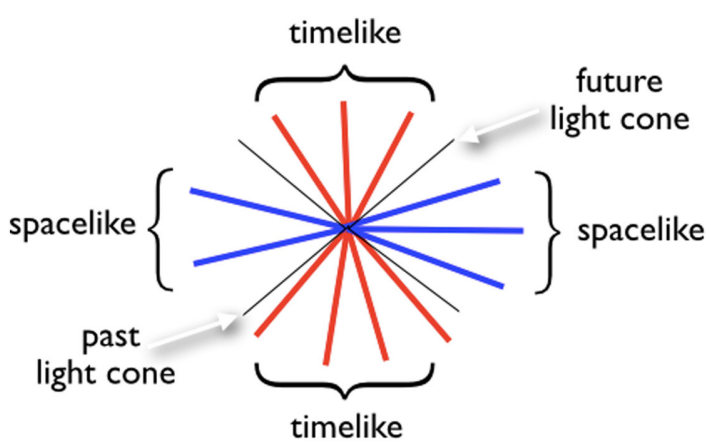

FIG. 3 (color online). Local causality implies the existence of one future and one past light cone at each point. The edges meeting at a vertex are always arranged in four groups of alternating type, time- or spacelike (with at least one edge in each group), as depicted. In the figure, time is pointing upwards, and the thin lines indicate the light cones located at the central vertex.

$$
\operatorname{vol}\left(\Delta_{\text {stt }}\right)=\frac{\sqrt{4 \alpha+1}}{4} a^{2}, \quad \operatorname{vol}\left(\Delta_{\text {sst }}\right)=\frac{\sqrt{\alpha(\alpha+4)}}{4} a^{2} .
$$

To be able to perform Monte Carlo simulations of the system, we analytically continue the parameter $\alpha$ to $-\alpha$ in the lower-half complex plane according to the usual CDT prescription [21]. The resulting real expression for the Wick-rotated regularized path integral in two dimensions is

$$
Z(\lambda)=\sum_{T} \frac{1}{C(T)} \mathrm{e}^{-\lambda a^{2}\left(N_{s t t} \frac{\sqrt{4 \alpha-1}}{4}+N_{s s t} \frac{\sqrt{\alpha(4-\alpha)}}{4}\right)}
$$

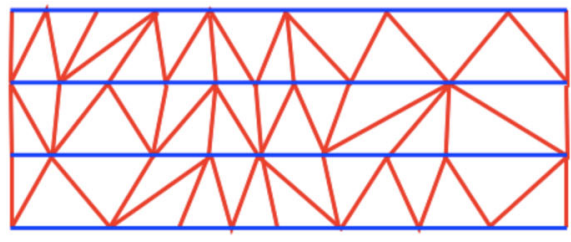

(a)
Note that for both triangle volumes to be positive, $\alpha$ must satisfy the inequality $1 / 4<\alpha<4$. The limiting value $\alpha=1 / 4$ corresponds geometrically to a collapse of the $s t$-triangles to zero volume, whereas for $\alpha=4$ the $s s t$-triangles are collapsed. In the isotropic case, $\alpha=1$, both triangles after Wick rotation are equilateral and identical. All LCDT simulations presented below were performed for $\alpha=1$.

Building blocks of the two triangle types are assembled into simplicial manifolds $T$ by "gluing" them together pairwise along boundary edges, where a timelike edge can only be glued to another timelike edge, and a spacelike edge only to another spacelike edge. Note that with respect to some overall flow of time, each of the two building blocks can appear with two different time orientations, which can be indicated by arrow assignments as illustrated in Fig. 2. By definition, all arrows are future pointing. Note that once a single triangle in a triangulation has been given a specific time orientation, the orientation of its neighbors, and of its neighbors' neighbors, etc. is also fixed, because the arrows on shared edges have to match.

Local causality is incorporated in the gluing rules by stipulating that before the analytic continuation there should be exactly one future and one past light cone at each interior point of the triangulation [14]. This condition is always satisfied at an interior point of a triangle, because up to diffeomorphisms the metric by construction is given by the Minkowski metric. It is also satisfied at points along edges where two triangles meet, unless the point happens to be a vertex, as can be seen by inspecting the geometry of the building blocks in Fig. 2. The requirement is only nontrivial at the vertices of the triangulation. When expressed in terms of the edges meeting at a vertex, it implies that they should come in four groups of alternating type (time- or spacelike) when going around the vertex once (Fig. 3), which imposes corresponding restrictions on the triangles meeting at the vertex.

The generic vertex structure in ordinary CDT, which uses only building blocks of type $\Delta_{\text {stt }}$, is also of this type, but by construction there is only a single spacelike edge each on the left and the right of the light cone, and the pair of these

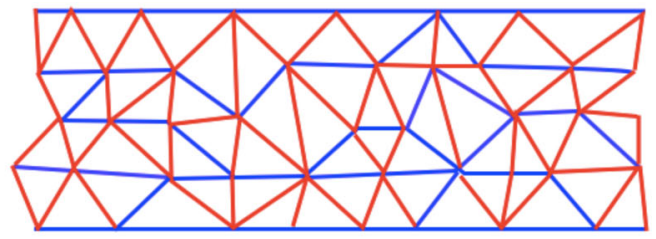

(b)

FIG. 4 (color online). Two strips of dynamically triangulated spacetimes with an initial (bottom) and a final spatial boundary (top). (a) In standard CDT only stt-triangles are used. There are two spatial edges meeting at each internal vertex, giving rise to the characteristic preferred proper-time slicing in terms of consecutive lines of spatial links. (b) LCDT works with both stt- and sst-triangles, allowing for a more general vertex structure, without preferred slicing. 
spacelike links forms part of a preferred slice of constant integer time. It is precisely the generalized vertex structure in locally causal DT that allows for configurations without this preferred time slicing. Fig. 4 illustrates the difference between a piece of causal DT and one of locally causal DT.

\section{PROPERTIES OF LCDT IN TWO DIMENSIONS}

Our task will be to investigate the properties of the path integral (4) in the continuum limit, where the sum is taken over an ensemble of triangulations of fixed topology, obeying local simplicial manifold conditions ${ }^{1}$ and with the vertex structure of locally causal DT described above. At this stage, there is no known exact solution of the continuum dynamics of LCDT quantum gravity in two dimensions; one difficulty is precisely the absence of a distinguished notion of time in terms of the lattice structure itself, which prevents the straightforward introduction of a transfer matrix used previously in solving CDT [8,15-17].

Since the configurations have two different kinds of edges, they can be thought of as a particular kind of twocolored graph, whose properties one may try to understand in a systematic way in the sense of enumerative combinatorics. The subgraph consisting of spacelike links only has the form of a stack of "bubbles," which on their inside are decorated with timelike links (see [14,22] for definitions and discussions of this substructure). The LCDT model may be supplemented by additional conditions, which restrict the type of (self)overlaps among these bubbles that are allowed. Since the bubbles are extended structures, these additional rules have a nonlocal character. They are motivated by the finding that for spatially compact boundary conditions in two dimensions the local condition of vertex causality described above does not imply global causality in the sense of the absence of (a specific class of) closed timelike curves (see Sec. V for an explicit example and further discussion). In turn, the presence of such curves is related to the presence of overlapping bubbles.

In our simulations both space and time will be compact. The topology of space will be a circle and time will be cyclically identified, which means that spacetime is topologically a torus $T^{2}$. Since we are primarily interested in "bulk" properties of the geometry, this choice is technically convenient: all vertices are interior vertices, on which vertex causality will be imposed, and the action is the one appearing in the path integral expression (4), without the need for adding any boundary terms.

The functional form of the Euclidean action, schematically given by $S=c_{1} N_{s t t}+c_{2} N_{s s t}$, for two positive constants $c_{1}, c_{2}$, is the most general one linear in "counting variables." These are the variables counting simplices of a particular dimension and type in a given triangulation $T$ : the numbers $N_{0}(T)$ of vertices, $N_{s}(T)$ of spacelike edges

\footnotetext{
${ }^{1}$ In dimension 2 , each internal edge is shared by exactly two triangles, and any two triangles share at most one edge.
}

and $N_{t}(T)$ of timelike edges, as well as the numbers $N_{s s t}(T)$ and $N_{s t t}(T)$ already introduced earlier. Our statement follows from the fact that these five variables are subject to three constraints,

$$
\begin{aligned}
N_{0}-N_{s}-N_{t}+N_{s s t}+N_{s t t} & =0, \\
N_{s s t}+2 N_{s t t}-2 N_{t} & =0, \\
N_{s t t}+2 N_{s s t}-2 N_{t} & =0,
\end{aligned}
$$

which must be satisfied on each configuration $T$ with torus topology.

We finally note that, at least in the absence of additional constraints on the bubble configurations, toroidal boundary conditions introduce a duality into the two-dimensional LCDT system. The duality transformation consists in swapping simultaneously the assignments timelike and spacelike of all edges in a given triangulation, which will convert all $s t$ t-triangles into $s s t$-triangles and vice versa. An admissible triangulation (one that satisfies the local conditions of a simplicial manifold and vertex causality) will under this transformation be mapped to another admissible triangulation with the same topology, with the roles of time and space interchanged. Of course, for $\alpha \neq 1$ the Boltzmann weights of a triangulation and its dual will in general be different.

\section{NUMERICAL SETUP}

We have used Monte Carlo techniques to sample the partition function or Euclideanized path integral (4) of locally causal dynamical triangulations, and compute expectation values of selected observables. An important ingredient is a set of Monte Carlo moves, which take the form of local changes in the simplicial geometry, and are designed to get us around the configuration space of the model by way of a Markov process. The four types of move we have worked with will be described below. Further technical details on their implementation may be found in [23]. Additional references on Monte Carlo moves in the context of causal dynamical triangulations are $[1,13]$.

The first type of move is a generalization of the $(0,2)$ move used in CDT, in which two adjacent stt-triangles that
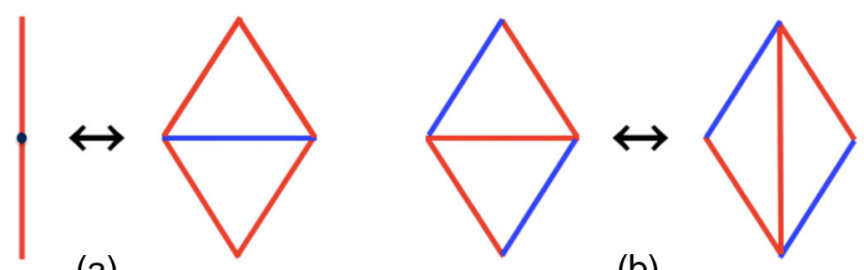

(b)

FIG. 5 (color online). Two types of Monte Carlo moves used in locally causal DT: (a) an example of a (0,2)-move and its inverse, also called a "link collapse"; and (b) an example of a (2,2)- or flip move and its inverse. 


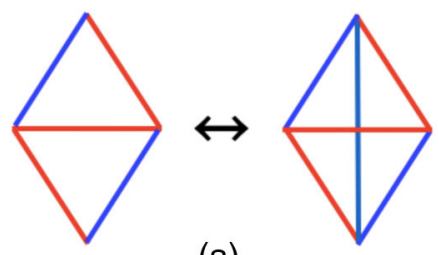

(a)

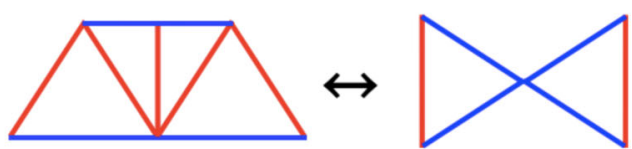

(b)

FIG. 6 (color online). The two remaining types of Monte Carlo moves used in locally causal DT: (a) an example of a (2,4)-move and its inverse; and (b) the pinching move and its inverse.

share a spacelike link are created simultaneously. The local starting configuration consists of a pair of timelike links, belonging to opposite sectors of a vertex (one link from inside the past and the other from inside the future light cone); see Fig. 5(a). This move is compatible with the time slicing of CDT geometries. Since this compatibility is no longer a requirement in locally causal DT, we also use the color-reversed counterpart of this move, where two adjacent $s s t$-triangles that share a timelike link are created from a pair of spacelike links, this time from opposite spacelike sectors of the light cone at the central vertex.

Also the second type of move, the $(2,2)$ - or flip move generalizes a local move already employed in CDT. It consists in flipping the diagonal inside a rhombus made of a pair of adjacent triangles. The version depicted in Fig. 5(b) is the one also permitted in CDT. In our simulations of locally causal DT, we in addition use the move with the opposite assignments of time- and spacelike edges. These are the only two flip moves compatible with vertex causality, if the character of the flipped edge remains unchanged. Two more flip moves are possible if the flipped diagonal link is allowed to change from time- to spacelike or vice versa.

Another type of move we have used in the simulations is a $(2,4)$-move, where the starting point is again given by a pair of adjacent triangles. A new configuration with identical boundary is obtained by "subdividing" the rhombus with another diagonal, thereby creating a four-valent vertex at the center; see Fig. 6(a) for an example. In order for vertex causality to be satisfied at the new vertex, the added diagonal has to be of opposite (time-/spacelike) type to the one already present. Eight variations of the $(2,4)$ move (and its inverse) are possible, depending on the type and orientation of the initial triangle pair, but six of them are equivalent to performing a $(0,2)$-move, which was already discussed above.

Lastly, we employ a "pinching" move, which is entirely new and not present in standard CDT, and was previously described in [13]. The initial local configuration for this move looks like a piece of regular CDT configuration, consisting of four stt-triangles forming a strip bounded above and below by line segments made out of spacelike links. The move pinches those two segments together in a single point, resulting in a pair of $s$ t-triangles, as illustrated by Fig. 6(b). In the simulations we also use the colorreversed version of this move.
In all cases, it is understood that whenever one of these moves is proposed by the computer algorithm, it will always be rejected if it violates either vertex causality or the simplicial manifold condition. The (overcomplete) set of these moves is likely to be ergodic, but we do not have a formal proof at this stage. The explicit proof may depend in subtle ways on the details of the ensemble, in particular, on excluding classes of bubble configurations associated with specific closed timelike curves that lead to unwanted global acausal behavior.

We have run several kinds of cross-check on the Monte Carlo simulations: first, that the acceptance rates of moves and their inverses are approximately the same, and secondly, that the frequency of occurrence for configurations with very small volume is compatible with the frequency predicted by the Boltzmann distribution. Lastly, our setup has an easy way to implement a CDT limit, which we can use to cross-check measured CDT results for the dynamical dimensions with the theoretical results available.

Note that apart from the flip move, all Monte Carlo moves described above alter the number of triangles in the triangulation, and therefore its two-volume. Since it is convenient from the numerical point of view to keep the total volume fixed, at least approximately, we use the standard DT prescription where the volume $V_{2}$ is allowed to vary in a narrow interval around a fixed target volume $V_{2}^{(0)}$. This is achieved by adding a quadratic term $\delta\left(V_{2}^{(0)}-\right.$ $\left.V_{2}\right)^{2}$ to the action, with a parameter $\delta>0$ determining the width of the interval. ${ }^{2}$ We tune the cosmological constant $\lambda$ such that the measured volumes are distributed symmetrically around $V_{2}^{(0)}$, and only collect data from triangulations that have precisely this target volume. The simulations are run such that there is about one sweep of length $L \approx 10^{6}$ between successive measurements.

As mentioned before, there is a straightforward way to obtain ordinary CDT simulations in our setup. It consists in setting $\alpha=1 / 4$ in the action (4), while maintaining the constraint $N_{2} \equiv N_{s s t}+N_{s t t}=$ constant. Since the term proportional to $N_{s t t}$ in the action now vanishes, stt-triangles can be created "at no cost" during the Monte Carlo simulation, while the number of $s s t$-triangles will diminish

\footnotetext{
${ }^{2}$ Note that for our standard choice $\alpha=1, V_{2}$ is proportional to the number $N_{2}$ of triangles.
} 
accordingly, thereby lowering the value of the Euclidean action. As a result, the triangulations quickly become pure CDT configurations, consisting only of building blocks $\Delta_{s t t}$. Below in Sec. VI we measure the Hausdorff and spectral dimensions of CDT quantum gravity. In addition to setting $\alpha=1 / 4$, we disable the $s s t$-triangles completely, to make sure that any fluctuations with nonvanishing $N_{s s t}$ are eliminated.

\section{CLOSED TIMELIKE CURVES}

For most of our measurements, the ensemble of locally causal triangulated geometries on which the dynamics takes place will consist of spacetimes of torus topology satisfying simplicial manifold conditions and vertex causality. However, for some purposes, when considering the time evolution of observables, it is convenient to restrict this ensemble further, because of the appearance of a particular class of closed timelike curves.

To explain this issue further, we introduce the notion of a timelike cycle in a locally causal triangulation. By this we mean a contiguous set of timelike links which together form a noncontractible loop of topology $S^{1}$, without self crossings or self overlaps (Fig. 7). In addition, whenever the loop passes through a vertex, the two timelike links of the loop meeting at the vertex must lie in opposite light cones, never inside the same (half of the) light cone. A spacelike cycle is defined analogously in terms of spacelike edges, and it is also required to be noncontractible. In the spacelike case, the cycle has to cross at each vertex from one spacelike sector outside the light cone to the opposite one.

In standard CDT in $1+1$ dimensions with its preferred time slicing [c.f. Fig. 4(a)], spacelike cycles only exist when space is compactified to a circle. In this case they simply coincide with the one-dimensional spatial slices at integer proper time. Likewise, timelike cycles only exist in CDT when time is compactified, in which case they can be

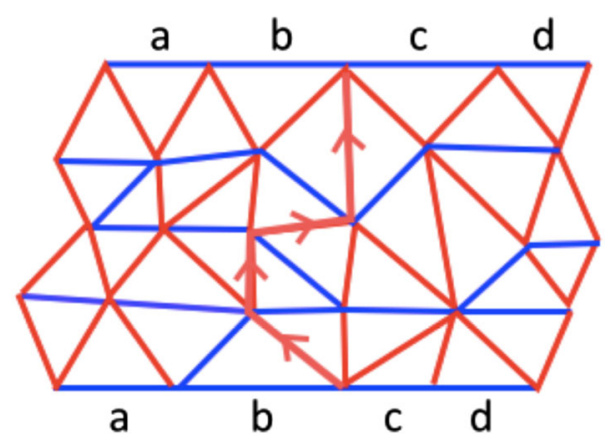

FIG. 7 (color online). A piece of LCDT, with an initial and a final spatial boundary (blue edges, bottom and top). When the two spatial boundaries are identified as indicated by the letters, the future-oriented timelike curve running from bottom to top (timelike edges with arrows) becomes a timelike cycle, as defined in the text.

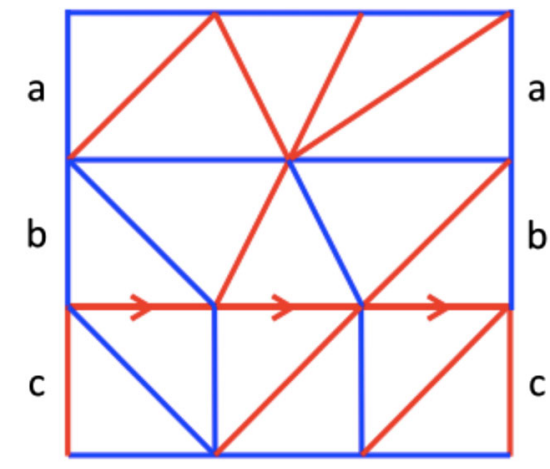

FIG. 8 (color online). A piece of locally causal DT, with an initial and a final spatial boundary (blue edges, bottom and top). Compactifying space as indicated by the letters results in a closed, future-oriented timelike curve (timelike edges with arrows), which intersects neither the initial nor the final boundary.

thought of as a particular ${ }^{3}$ lattice realization of closed timelike curves. As a consequence of how they traverse the light cones at vertices, they are also time oriented, either in positive or negative time direction. As usual, the reason for compactifying time in CDT simulations is merely one of convenience, and the appearance of closed timelike curves is an inevitable side effect, which is not expected to have much influence on the measurement of bulk observables like the dynamical dimensions considered below. ${ }^{4}$

The closed timelike curves we are primarily concerned about in LCDT are not the ones winding around the compactified time direction, but around the spatial direction, and which would still be present if spacetime was a cylinder $I \times S^{1}$ (with compact spatial slices) instead of a torus; see Fig. 8 for an example. In the remainder of this work, when we talk about closed timelike curves (CTCs), we will mean only this class of timelike cycles. It turns out that local vertex causality does not preclude the presence of these curves, although it does prevent the occurrence of contractible timelike loops [22]. One way of finding them is by running an algorithm that assigns time labels to vertices of a given locally causal DT. Of course, an explicit choice of time has to be made in the LCDT model, because-unlike in usual CDT-there is no preferred lattice substructure one can refer to as a natural time label.

The prescription for assigning time labels to vertices in a given geometry we have used in the present work is to pick a spacelike cycle in the geometry and define it as "space at time $t=0$." Vertices lying in the future of this slice are then

\footnotetext{
${ }^{3}$ They are particular in the sense that one could also consider paths that run not only along the edges of a triangulation, but also through the interiors of triangles.

${ }^{4}$ Obviously, in any concrete implementation the dependence of observables on boundary conditions and other finite-size effects should always be monitored. Although CTCs are unphysical classically, their inclusion in the regularized path integral does not a priori imply unphysical behavior of the final continuum theory.
} 


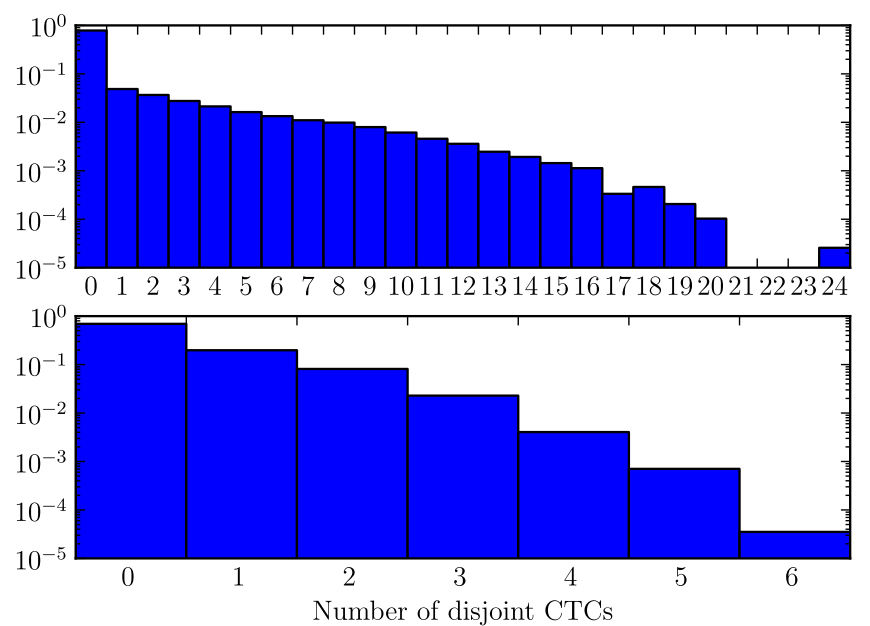

FIG. 9 (color online). The frequency of disjoint timelike loops in 48.000 sweeps. The upper histogram is for $N_{2}=10.000$ and the lower one for $N_{2}=100.000$.

successively assigned time labels, which are computed as the average distance of the vertex $v$ to the initial slice along any oriented timelike path from the slice to $v$ (see [23] for more details on the algorithm). The distance along any given path is simply given by the number of timelike links it contains, the so-called link distance. Note that the time label of a vertex will in general not be an integer. Once the vertices are labeled, one can in a straightforward way also associate time labels with edges or more extended regions like spacelike cycles by averaging over the time labels of the vertices contained in them. In standard CDT, this prescription reproduces the usual integer proper-time slicing.

The algorithm implementing the vertex labeling breaks down when it encounters a CTC, like that depicted in Fig. 8, because for any vertex lying on the curve or in its future there will be infinitely many timelike paths connecting it to the initial slice. We conclude that in LCDT, at least when space is compactified, local causality does not imply global causality in the sense of an absence of CTCs.

Whether or not the presence of closed timelike curves has any consequences for the continuum limit of the model is a priori unclear. We have performed a number of measurements to get a better quantitative idea of how many CTCs there are, depending on the size of the triangulation. Figure 9 shows two histograms of the frequencies of disjoint CTCs (CTCs without mutual overlap $^{5}$ ). We observe that the typical number of disjoint closed timelike curves present in a given configuration goes down significantly when the volume is increased tenfold from $N_{2}=10.000$ to $N_{2}=100.000$ triangles. On the other hand, the ratio of triangulations

\footnotetext{
${ }^{5}$ These curves form a subclass of CTCs; the number of all CTCs can be considerably larger, especially when there are many disjoint CTCs.
}

which contains any CTCs at all increases from about $21 \%$ to $30 \%$. We therefore have no indication that CTCs disappear completely as the volume grows. One contributing factor is presumably that it takes much longer to break up a CTC by a local Monte Carlo move when the CTCs become highly diluted in a triangulation.

\section{OBSERVABLES}

Having introduced both the theoretical and numerical setup of locally causal dynamical triangulations in $1+1$ dimensions, we now discuss the measurements of several observables in this model of quantum geometry, concentrating on the isotropic case $\alpha=1$. Our main aim is to understand whether the model's continuum limit coincides with that of either Euclidean or causal dynamical triangulations in two dimensions. It would be exciting if LCDT constituted a new universality class, but this seems a priori less likely because of the apparent scarcity of universality classes among two-dimensional models of pure geometry without matter coupling.

If LCDT quantum gravity were to lie in one of the two known universalities of DT models in two dimensions, our best guess at this stage would be that it is equivalent to CDT, for two reasons: first, there is good evidence that this is true in three spacetime dimensions [14], in the sense that there one finds in both models a phase whose ground state has the scaling properties of a Euclidean de Sitter universe. (Of course, this by no means constitutes a proof that the same happens in two dimensions, which differs in terms of both its geometric degrees of freedom and its phase structure.) Second, the difference between DT and CDT in two dimensions has so far been explained in terms of the absence of baby universes in the latter $[10,24]{ }^{6}$ Since the condition of vertex causality in locally causal DT suppresses the light cone degeneracies characteristic of topology change and therefore of the creation of baby universes, the LCDT model seems closer to CDT than to DT, where baby universes dominate.

\section{A. Volume profile}

We begin by examining the so-called volume profile of a typical geometric configuration generated by the Monte Carlo simulation of LCDT. The volume profile is simply given by the size of the spatial volume as a function of time. Since by construction the model does not have a distinguished notion of time in terms of some lattice substructure, we make use of the notion of time introduced in Sec. V above. For this purpose, a spatial slice at fixed time is any spacelike cycle-as defined at the beginning of Sec. V-and its time label is obtained by averaging over the

\footnotetext{
${ }^{6}$ More precisely, it is the absence of the possibility for baby universes to proliferate without limit; a limited, controlled presence of baby universes is compatible with two-dimensional CDT, as demonstrated by the model of generalized CDT $[18,19]$.
} 


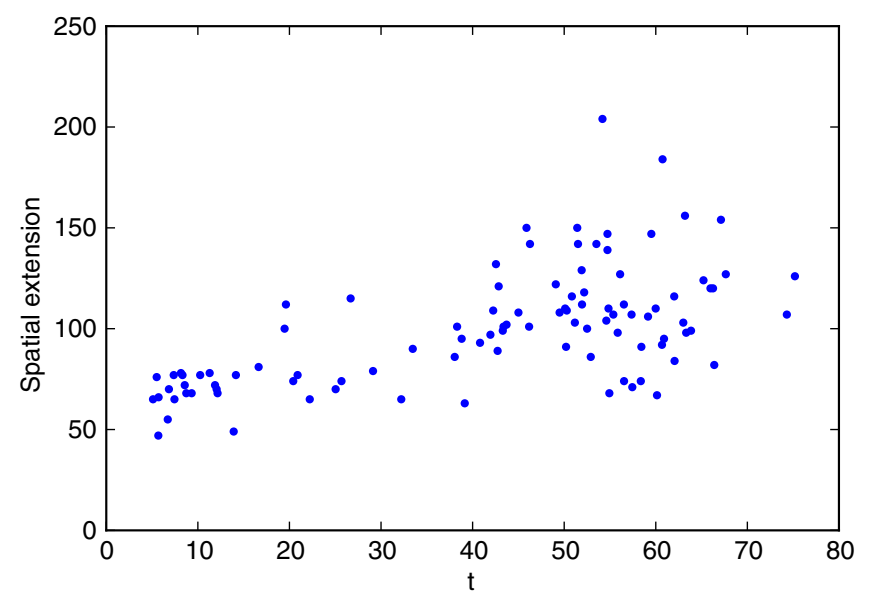

FIG. 10 (color online). Sampled LCDT volume profile for $N_{2}=10.000$, where $t$ denotes the time label of a spacelike cycle and the discrete spatial extension is the number of spacelike links in the cycle at a given $t$.

time labels of all of its vertices. The volume of a spatial slice is the number of links contained in it. To determine the complete volume profile of a spacetime configuration, one has to identify all of its spatial cycles, which is a nontrivial task. Recall that unlike in CDT, in LCDT different spatial cycles can cross and overlap along some subset of spatial edges.

For simplicity, we have considered sampled volume profiles instead of complete ones; for a geometry of volume $N_{2}=10.000$ we have randomly sampled 100 spatial slices, and for each slice determined its volume and time label. Figure 10 shows one such sample, to illustrate the situation. We note that some time labels are very close to each other, which indicates that they probably share one or more spacelike links. The sample shows large volume fluctuations within small time intervals, without any discernible overall shape. This finding is only qualitative, but it is compatible with the typical, strongly oscillating volume profiles encountered in simulations of two-dimensional CDT [25].

\section{B. Behavior of minimal loops}

A phenomenon that will potentially affect the measurement of dimensions discussed below is the overall shape of the toroidal configurations. We saw in the previous subsection that their volume profiles seem to be strongly fluctuating, and are comparable to what one finds in twodimensional CDT quantum gravity. Large fluctuations are commonplace in two dimensions, because there is only a single coupling constant (the cosmological constant) which sets the scale of both the spatial universe and its quantum fluctuations [8]. ${ }^{7}$ This is in line with the fact that general relativity in two dimensions is trivial.

\footnotetext{
${ }^{7}$ The unique length scale of the quantum theory is $\Lambda^{-1 / 2}$, where $\Lambda$ is the dimensionful, renormalized cosmological constant.
}

A new feature in LCDT is the variable length of the configurations in the time direction, since by construction the fixed time slicing is absent. As a result, both the spatial extension of the Universe and its time extensiondetermined by the prescription used for measuring the volume profile, say-will evolve dynamically. For example, the torus may become very thin in one of its directions, an effect which may be quantified by monitoring the length of closed noncontractible curves. While in CDT individual slices of constant time can become very short (the minimal length of a spatial $S^{1}$ compatible with manifold conditions is attained by cycles of three links), the probability for this to happen can be made very small by choosing the total time extent $t_{\text {ТОТ }}$ and the total volume $N_{2}$ suitably. By contrast, in LCDT it can in principle happen that the torus becomes uniformly thin in one of its directions, even for large $N_{2}$, if this is dynamically preferred.

The relevance of this for the measurement of dynamical dimensions is the possible appearance of finite-size effects, even when the total volume is large. For example, this happens when the paths of random walkers-used to determine the spectral dimension-start winding around the torus more than once. To obtain an estimate of the size of this effect we have set up an algorithm which searches the tori for minimal noncontractible loops. It does not distinguish between time- and spacelike links, which implies that the minimal loops found can be made up of any link types.

The algorithm consists of the following steps: on a given LCDT configuration $T$, pick a vertex $v$. Starting at $v$, perform a breadth-first search until the area searched starts to self overlap at some other vertex $v^{\prime}$. Next, determine whether the minimal closed curve $c$ through $v$ and $v^{\prime}$ obtained in this way is contractible or not, by starting breadth-first searches on either side of $c$. If those searches start overlapping, $c$ is a noncontractible closed curve through $v$ of minimal length and we record its length. Because this procedure is rather costly in computational terms, we do not repeat it for every vertex of $T$, but only for a sample of 1.000 randomly chosen initial vertices on $T$. For a given triangulation $T$ we therefore end up with a sample of locally minimal loops (locally because they pass through prescribed vertices on the torus).

To obtain Fig. 11, we have performed the sampling of minimal loops at each step during the thermalization of a Monte Carlo simulation of 280 time steps, corresponding to 280 sweeps, for a triangulation size $N_{2}=100.000$. At each step, we plot only the shortest and longest minimal loop length of the sample; all other minimal loop lengths lie in between these two values. Since our samples are quite large, the lower curve is probably a good indicator of the global minimum of the length of noncontractible loops on $T$, which roughly speaking lies in the range $20-35$. This is far away from the kinematically allowed minimum of 3 and shows that the torus does not become very thin in 


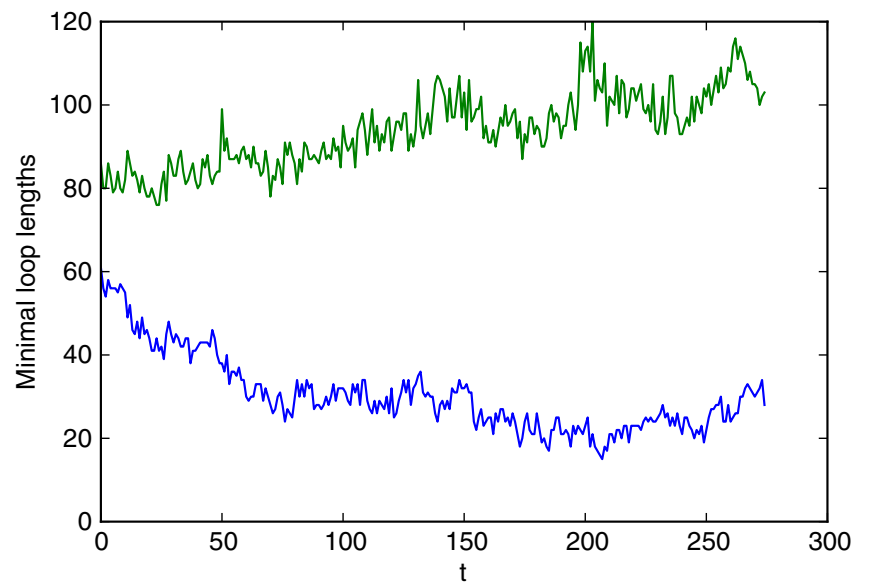

FIG. 11 (color online). Evolution of the shortest and longest loop lengths from a sample of minimal noncontractible loops through randomly chosen vertices on a given triangulation, as a function of Monte Carlo time $t$, and at discrete volume $N_{2}=100.000$. The largest $t$-values are just before the onset of thermalization.

some places. ${ }^{8}$ In addition, the fact that there is a significant distance between the upper and lower curves shows that the torus does not degenerate by becoming very long in one direction and uniformly short in the other. On the other hand, a triangle count of $N_{2}=100.000$ according to (4) at $\alpha=1$ corresponds to a volume $V=\sqrt{3} N_{2} / 4 \approx 43.300$, where we have set the lattice constant to unity, $a=1$. Assuming the two torus directions are approximately of equal length, this corresponds to an average linear extension of the torus in the range of 150-200, of which the shortest loop length therefore is only a small fraction. It implies that fluctuations in the linear extension of the LCDT configurations are large, even if the total volume is also large. We comment further on this characteristic feature of two-dimensional quantum gravity in Sec. VID below.

The overall conclusion is that at system size $N_{2}=$ 100.000 finite-size effects for observables involving shortest (geodesic) distances should not play a role at least up to link distances of about 30, and for observables involving closed random walks at least up to about 500 steps. ${ }^{9}$ Looking ahead to the measurement of dimensions presented below, this still leaves plenty of room for finite-size effects on larger scales, but they are not quantified easily above the thresholds just mentioned.

\footnotetext{
${ }^{8}$ Note that our algorithm does not determine which of the torus directions any particular minimal loop winds around. Also in this respect the information is distinct from that contained in volume profiles of the kind shown in Fig. 10.

${ }^{9}$ Note that we are not making a distinction between link distance on the triangulated lattice and link distance on the dual lattice. We have not determined the relative scale between these two notions of geodesic distance on typical LCDT configurations. The numbers given in the text should therefore be treated only as rough estimates.
}

\section{Spectral dimension}

Dynamical dimensions, like the spectral and Hausdorff dimension, are important and popular examples of observables in models of nonperturbative quantum gravity because of their computational accessibility in many different contexts. A key insight is that the values of these dimensions do not have to coincide with the dimensionality of the triangular building blocks used to construct the regularized model if one takes a nontrivial, infinite continuum limit, as we are doing. Furthermore, a familiar feature from studying graphs and fractals, namely, the fact that there exist "spaces" of noninteger dimension, is also encountered in systems of dynamical triangulations. This is not necessarily inconsistent from a physical point of view as long as the anomalous values of the dimensions are confined to a highly quantumfluctuating, nonsemiclassical regime, typically at the Planck scale. Since there is no nontrivial classical theory of two-dimensional general relativity whose solutions might be recovered from a corresponding quantum theory in the limit as $\hbar \rightarrow 0$, there are no a priori physicality constraints on the Hausdorff and spectral dimension of an ensemble ${ }^{10}$ of DT configurations in two dimensions, causal or otherwise.

For Euclidean DT in two dimensions, from theoretical scaling arguments the spectral dimension is 2 and the Hausdorff dimension 4 [26], which has also been corroborated numerically [27]. Invoking an equivalence between CDT configurations and tree graphs, CDT in $1+1$ dimensions can be shown to have a spectral dimension of at most 2 and a Hausdorff dimension of almost surely 2 [28], the latter in agreement with earlier theoretical $[8,10]$ and numerical [25] results. In the context of LCDT, we first investigate the spectral dimension. In the section following this one, we examine the Hausdorff dimension, which appears to be the quantity best suited to discriminating between the different universality classes.

The first step in measuring the spectral dimension is to define a discrete diffusion process on a two-dimensional Euclideanized locally causal triangulation $T$. This takes the form of a random walk moving in steps of unit distance between the centers of neighboring triangles as a function of a discrete external diffusion time $\sigma$, analogous to what was done in CDT in four dimensions [29,30]. In other words, the diffusion takes place along the edges of the trivalent lattice dual to $T$. Calling $K_{T}\left(i, i_{0} ; \sigma\right)$ the probability to go from triangle $i_{0}$ to triangle $i$ in $\sigma$ steps, satisfying $\sum_{i} K_{T}\left(i, i_{0} ; \sigma\right)=1$, the discrete diffusion equation on the triangulation $T$ reads

\footnotetext{
${ }^{10}$ When talking about "the Hausdorff dimension," say, of (C)DT, we mean of course the expectation value of this quantity, measured in the ground state of the relevant ensemble.
} 


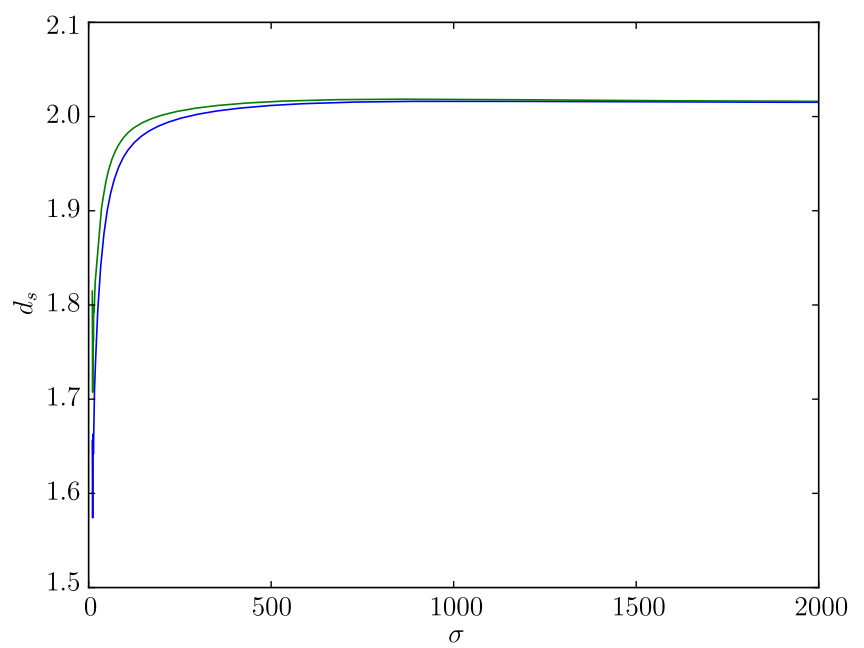

FIG. 12 (color online). The spectral dimension of CDT as a function of the diffusion time $\sigma$, measured at volume $N_{2}=100.000$, and time extension $t_{\mathrm{TOT}}=80$. The upper, green line is the dimension $d_{s}^{(1)}$ of Eq. (9) and the lower, blue line is the dimension $d_{s}^{(2)}$ of Eq. (10). Statistical error bars are too small to be displayed.

$$
\begin{aligned}
& K_{T}\left(i, i_{0} ; \sigma+1\right) \\
& \quad=(1-\chi) K_{T}\left(i, i_{0} ; \sigma\right)+\frac{\chi}{3} \sum_{j \text { n.n. of } i} K_{T}\left(j, i_{0} ; \sigma\right),
\end{aligned}
$$

subject to the initial condition $K_{T}\left(i, i_{0} ; \sigma=0\right)=\delta_{i, i_{0}}$. The sum on the right-hand side of (6) is over the three nearest neighbors $j$ of triangle $i$, and $\chi \in[0,1]$ is a diffusion constant which allows for a nonvanishing probability $(1-\chi)$ that the random walker remains at the same triangle during a diffusion step. It is included merely for convenience, to somewhat smoothen out the discretization artifacts for short diffusion paths. In particular, there is an asymmetry between paths of even and odd numbers of steps (c.f. the discussion in [29]), with a corresponding oscillatory behavior in the curve for $d_{s}$ that is also present in our Figs. 12-13 when one zooms into the region below $\sigma \approx 50$. A diffusion constant $\chi<1$ has been used previously when studying the spectral dimension in three-dimensional CDT [31]. In our simulations, we have worked with $\chi=0.8$ throughout.

To extract the spectral dimension, we consider closed random walks, beginning and ending at a specified triangle $i_{0}$. They enter into the calculation of the average return probability

$$
P_{T}(\sigma)=\frac{1}{N_{2}(T)} \sum_{i_{0} \in T} K_{T}\left(i_{0}, i_{0} ; \sigma\right)
$$

for a given triangulation $T$. The spectral dimension $d_{s}$ is obtained from the expectation value $\langle P(\sigma)\rangle_{N_{2}}$ of the observable (7) in the ensemble of triangulations of fixed volume $\mathrm{N}_{2}$ according to

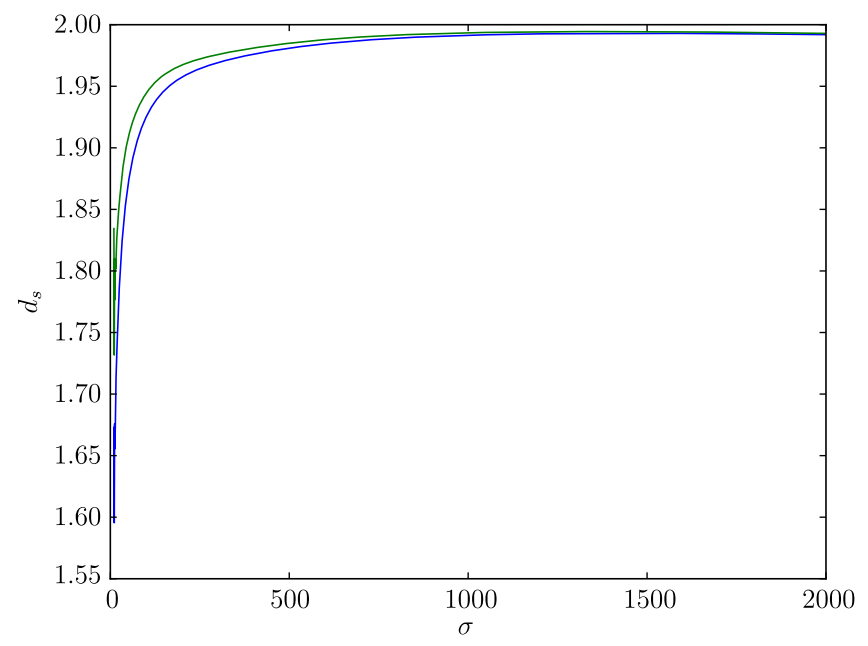

FIG. 13 (color online). The spectral dimension of locally causal DT (with $\alpha=1$ ) as a function of the diffusion time $\sigma$, measured at volume $N_{2}=100.000$. The upper, green line is the dimension $d_{s}^{(1)}$ of Eq. (9) and the lower, blue line is the dimension $d_{s}^{(2)}$ of Eq. (10). Statistical error bars are too small to be displayed.

$$
d_{s}(\sigma):=-2 \frac{\mathrm{d} \ln \langle P(\sigma)\rangle}{\mathrm{d} \ln \sigma} .
$$

In practice, we perform a "double" random sampling, where for each randomly chosen triangulation we pick ten times a triangle randomly as starting point $i_{0}$ for a random walk, and then repeat the process for at least 400 triangulations. For a diffusion process on classical, flat IR ${ }^{d}$, the formula analogous to (8) simply reproduces the topological dimension $d$, independent of $\sigma$, but in the quantum context the behavior of $d_{s}$ can be more complicated and, generally speaking, $\sigma$ dependent. By not denoting the $N_{2}$ dependence in (8) explicitly we mean to indicate that $d_{s}$ is determined in the limit of large volumes where this dependence gradually disappears.

When the total spacetime is compact, the spectral dimension will always go to zero for sufficiently large $\sigma$. This is a finite-size effect which occurs when generic random walks become sufficiently long to wrap around space one or more times. We are primarily interested in the $\sigma$-regime below this range. If the system develops a stable plateau below the scale where significant finite-size effects kick in, we refer to this constant value of $d_{s}$ as the spectral dimension of the underlying quantum spacetime. Note that if the system size is too small, a plateau will never form due to a dominance of finite-size effects.

Since there are no published numerical results on the spectral dimension of two-dimensional CDT, and since it will be useful to have a point of reference for the measurements in LCDT, we first present our results for the spectral dimension of regular CDT quantum gravity on a two-torus. As explained earlier, the reduction to pure CDT configurations is achieved by setting $\alpha=1 / 4$ in the action. To understand better the effects of the discretization, 
we have used two different discrete versions of the defining formula (8) for the spectral dimension. Having determined the expectation value $\langle P(\sigma)\rangle$ for integer $\sigma$ from the data, we have employed two different implementations in terms of finite differences. The standard choice is

$$
d_{s}^{(1)}=-2 \frac{\ln \langle P(\sigma+1)\rangle-\ln \langle P(\sigma)\rangle}{\ln (\sigma+1)-\ln \sigma} .
$$

In addition, we have used the alternative form

$$
d_{s}^{(2)}=-2 \sigma\left(\frac{\langle P(\sigma+1)\rangle}{\langle P(\sigma)\rangle}-1\right),
$$

which has the same continuum limit and the advantage that no expensive functions are required.

The CDT results for the spectral dimension $d_{s}$ are displayed in Fig. 12, for data taken at volume $N_{2}=$ 100.000 and time extension $t_{\text {TOT }}=80$. For $\sigma \lesssim 700$ there is a small discrepancy between the curves corresponding to the two different discretizations, giving us an estimate of the systematic error of determining $d_{s}$ for small values of $\sigma$. For larger $\sigma$, both curves merge into what is essentially a single plateau. The spectral dimension of CDT extracted from data on the plateau is $d_{s}=2.02 \pm 0.02$, in good agreement with the expected value of 2 .

The curves for the spectral dimension for LCDT are shown in Fig. 13. Qualitatively the plot is similar to that of CDT, but the plateau is reached only for somewhat larger diffusion times $\sigma \gtrsim 1.000$. The numerical result for the spectral dimension is $d_{s}=1.99 \pm 0.02$, which we regard as a convincing confirmation that the spectral dimension of locally causal DT is 2, like that for DT and CDT quantum gravity.

\section{Hausdorff dimension}

The Hausdorff dimension $d_{h}$ is a key quantity to discriminate between distinct universality classes of twodimensional DT quantum gravity. The general idea is to relate the volume $V$ of compact, connected regions in space-typically discrete analogues of geodesic balls around a chosen point - to their linear size, e.g. the radius $r$ of the region, and to extract the leading scaling behavior from $\langle V(r)\rangle \sim r^{d_{h}}$. For our purposes, we will use a "differential" version of this relation, where one monitors the onedimensional volumes of spherical shells around a given triangle $i_{0}$ or, equivalently, the number of (dual) vertices at radial distance $r$ from a vertex $i_{0}$ of the lattice dual to a given LCDT configuration $T$. We define $n\left(r, i_{0}\right)$ as the number of triangles found at geodesic distance $r$ from $i_{0}$, where geodesic distance is defined as the (integer) length of the shortest path along edges of the dual lattice. We have $n\left(0, i_{0}\right)=1$ and $n\left(1, i_{0}\right)=3$ for all $i_{0}$, because each triangle has exactly three neighbors and therefore the dual lattice is trivalent. Every triangle of $T$ will appear in exactly one of the shells, implying that $\sum_{r} n\left(r, i_{0}\right)=N_{2}(T)$. The identification of the shells can be implemented as a modified breadth-first search, which keeps track of when a change of shells occurs. Averaging over all initial triangles $i_{0} \in T$, we obtain the average shell volumes at radius $r$,

$$
n(r)=\frac{1}{N_{2}} \sum_{i_{0}=1}^{N_{2}} n\left(r, i_{0}\right) .
$$

In what follows, we refer to the function $n(r)$ as the shape of a triangulation.

To extract the Hausdorff dimension, we have applied finite-size scaling to the expectation value $\langle n(r)\rangle$ of the shape function (11). ${ }^{11}$ The simulations consisted of 48.000 sweeps each at volumes $N_{2}=100.000,200.000,300.000$ and 400.000, and were done for LCDT (at $\alpha=1$ ) and, for reference and comparison, also for CDT (corresponding to $\alpha=1 / 4)$. The scaling ansatz for the radius and shell volume is $r \rightarrow x=N_{2}^{-1 / d_{h}} r$ and $\langle n(r)\rangle \rightarrow N_{2}^{-1+1 / d_{h}}\langle n(r)\rangle$ respectively. Individual data points $\langle n(r)\rangle$ were transformed into curves via spline interpolation and a Levenberg-Marquardt least-square fit was used to align the shapes [23].

In maximizing the overlap we have taken into account all $x$-values where $\langle n(r)\rangle$ has at least half of its maximal value. We have also measured the short-distance Hausdorff dimension for small $x$ by optimizing the overlap of the initial rising slopes of the curves $\langle n(r)\rangle$. In principle this dimension need not coincide with the global Hausdorff dimension $d_{h}$ we have been considering [32], but in our case there was little difference. Our results for the best overlap of the shape functions for the CDT case are shown in Fig. 14; they correspond to a Hausdorff dimension $d_{h}=2.2 \pm 0.2$, which is compatible with the known value of $2 .{ }^{12}$ Our error bars are rather large, because the best fit depends quite sensitively on the $x$-range for which the overlap is optimized.

The analogous data for LCDT are displayed in Fig. 15. Maximal overlap is achieved for a Hausdorff dimension $d_{h}=2.71 \pm 0.2$, which is far away from our conjectured CDT value of 2, and even further away from the DT value of 4 . We conclude that LCDT very likely lies not in the same universality class as DT. Contrary to our expectation, equivalence of LCDT and CDT appears to be excluded too. Instead, our measurements point towards LCDT lying in a new universality class, not hitherto seen in quantum models

\footnotetext{
${ }^{11}$ We also tried to extract the Hausdorff dimension from the scaling relation $\bar{r}\left(N_{2}\right) \sim N_{2}^{1 / d_{h}}$ for the average linear extension $\bar{r}$ defined in Eq. (13), but this did not yield meaningful results because of the convergence issues to be described in more detail below.

${ }^{12}$ For CDT in two dimensions, the Hausdorff dimension has been measured previously [25], with good results, from finite-size scaling of the distribution of spatial volumes. Because of the absence of a predefined time function, this method is not computationally feasible for LCDT.
} 


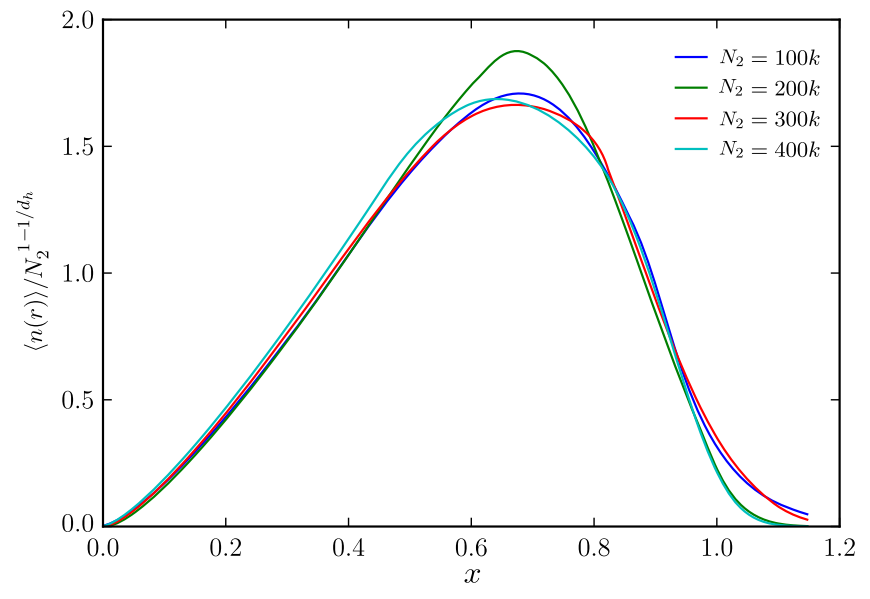

FIG. 14 (color online). CDT quantum gravity: Fit for best overlap of the rescaled shapes $\langle n(r)\rangle / N_{2}^{-1+1 / d_{h}}$ as a function of the rescaled distance $x=r / N_{2}^{1 / d_{h}}$, for Hausdorff dimension $d_{h}=2.2$. The extension in time direction was set to $t_{\mathrm{TOT}}=80$.

of two-dimensional pure gravity. This would be a truly interesting result, and it warrants another critical look at the strength of our evidence.

As is apparent from Figs. 14-15, the quality of the overlaps is not very good. Could there be systematic sources of error that affect our results to the extent that they ultimately are not in contradiction with $d_{h}=2$ for LCDT? In other words, may we be underestimating our error bars significantly? It may be worth recalling that it took some time to nail down the Hausdorff dimension of two-dimensional DT quantum gravity numerically. In the words of the authors of [33], early simulation results were "remarkably inconclusive" (see [33] for further references). The same work also used finite-size scaling of the shape function to determine $d_{h}$, with a fit quality somewhat similar to ours. Especially when using the dual lattice-as

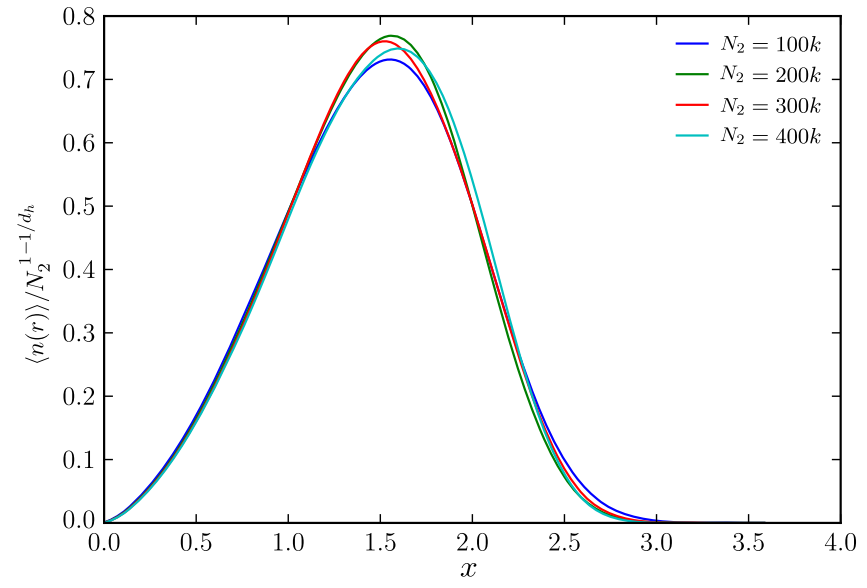

FIG. 15 (color online). LCDT quantum gravity: Fit for best overlap of the rescaled shapes $\langle n(r)\rangle / N_{2}^{-1+1 / d_{h}}$ as a function of the rescaled distance $x=r / N_{2}^{1 / d_{h}}$, for Hausdorff dimension $d_{h}=2.7$. we are also doing in the present work-the Hausdorff dimension extracted this way was significantly off the mark $\left(d_{h}=3.15\right.$ instead of the known, correct value 4$)$. Of course, one should keep in mind that these simulations were performed for a geometric ensemble different from LCDT and for moderate lattice sizes $N_{2} \leq 32.000$ only. On the other hand, the causal gluing rules of LCDT introduce a local "stiffness" in the configurations compared to DT, which is likely to require larger volumes to achieve numerical results of comparable quality.

In the case of DT simulations, significant progress with respect to the convergence of fits was obtained by introducing a "phenomenologically fudged" scaling relation for the geodesic distance [27], namely,

$$
x=\frac{r+a}{N_{2}^{1 / d_{h}}+b},
$$

where $a$ and $b$ are two parameters meant to compensate lattice artifacts at short distances. We have also tested relation (12), but found that nonvanishing values for $a$ and $b$ steer the CDT results even further away from 2 and also increase the sensitivity to the choice of fitting region.

A generic feature of two-dimensional quantum gravity illustrated by the numerical difficulties already mentioned is the fact that in two dimensions quantum fluctuations are always large, even for large lattice volumes. This is different from CDT in higher dimensions, say, where the dynamics is governed by two scales: one macroscopic, related to the overall size of the Universe, and another one microscopic, setting the scale of quantum fluctuations. In two dimensions, there are no nontrivial classical solutions, and there is only a single scale, that of the quantum fluctuations.

In this situation, it is therefore natural for finite-size effects to be generically large, especially when there are noncontractible directions along which space can become small, as can happen for the torus topology used for LCDT. ${ }^{13}$ This is certainly relevant when measuring the Hausdorff dimension; when the geodesic balls centered at some triangle $i_{0}$ start wrapping around one of the torus directions, the interpretation of the scaling relation by which we extract $d_{h}$ will be affected, in the sense that only triangles not visited previously will be counted as belonging to a given radial shell. Of course, one can keep extracting the Hausdorff dimension regardless, but should be aware that it contains also global, topological information.

To get further insights into the origin of the relatively poor quality of our fits, we have measured yet another observable, the average linear extension [29]

$$
\bar{r}=\frac{1}{N_{2}} \sum_{r} r \cdot n(r)
$$

\footnotetext{
${ }^{13}$ Note that the DT simulations mentioned above use the topology of a two-sphere. Also this indicates the need to go to larger volumes in the LCDT case.
} 
of a given triangulation $T$ of discrete volume $N_{2}$, which is just the weighted average of the geodesic distance $r$. As is described in more detail in the appendix, the observable $\bar{r}$ has convergence issues, which appear to persist even on large lattices and after a large number of sweeps. What seems to happen to the geometrical configurations is that most of the time they are approximately "square shaped," with comparable linear extensions for either torus direction, but every so often make an excursion to an overall shape that is elongated, where one torus direction becomes longer than the other one, with $\bar{r}$ increasing as a result. After a relative maximum of the two lengths has been reached, the system gradually reverts back to being square shaped and stays there for a while before another excursion takes place (see Fig. 16 for illustration).

Of course, square and elongated configurations (for identical volume $N_{2}$ ) not only have different average extensions $\bar{r}$, but also different shape functions $n(r)$ (see the appendix) and therefore in general different Hausdorff dimensions. A likely explanation for our inaccurate determination of $d_{h}$ is therefore the failure of the shape to stabilize during the course of the simulation, and the finite-size effects associated specifically with elongated shapes, in addition to the already mentioned large magnitude of the quantum fluctuations overall. This is supported by a numerical experiment we have performed in pure CDT quantum gravity at a volume $N_{2}=9.000$. For $t_{\mathrm{TOT}}=80$ time slices, the behavior was "squarelike," in the sense that the Monte Carlo history of the average extension $\bar{r}$ did not have any peaks. However, when we shortened the time extension to $t_{\text {TOT }}=20$, peaks similar to those depicted in Fig. 16 appeared.

Lastly, in our search for ways to improve the convergence behavior of LCDT quantum gravity, we investigated what happens when self-overlapping bubbles are not allowed to

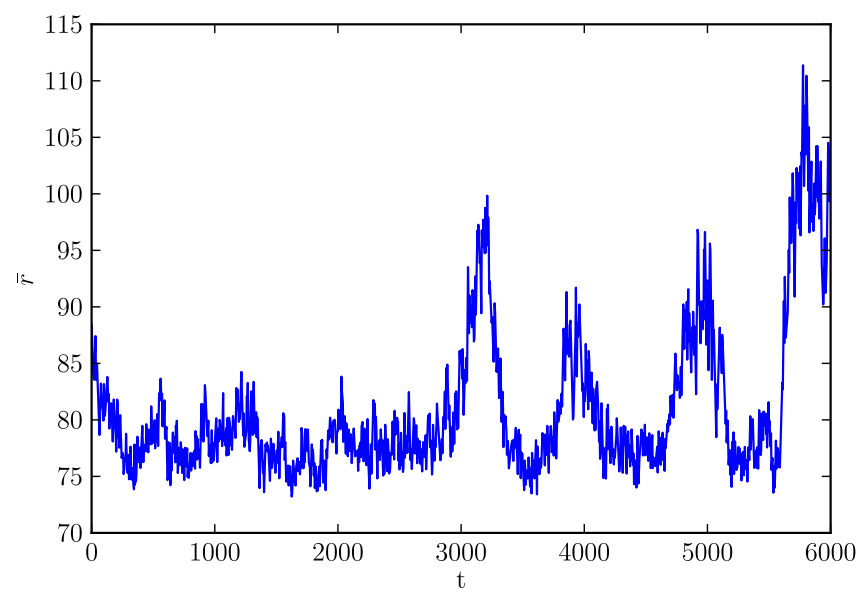

FIG. 16 (color online). Development in Monte Carlo time $t$ of the average linear extension $\bar{r}$ of a LCDT configuration $T$ of volume $N_{2}=50.000$. Isolated peaks in $\bar{r}$ keep occurring, even as the number of sweeps becomes very large. (Note that the y axis has an offset of 70.) occur. We mentioned these structures briefly in Sec. III above. A bubble is a contractible loop of spacelike links, which in its interior is decorated by timelike links only (Fig. 17). It always has two $s s t$-triangles at its end points and consists of stt-triangles otherwise. When a bubble winds around a compact torus direction, it can touch itself again (self overlap) along one or more spacelike edges of its boundary (see Fig. 18 for a simple example). Self-overlapping bubbles are geometrically significant, becausedepending on their interior geometry - they can give rise to timelike cycles as defined at the beginning of Sec. V. Their appearance is not forbidden by local vertex causality. Relevant to our present discussion is the fact that globally self-overlapping bubbles cause severe thermalization issues in $2+1$ dimensions, and therefore were removed from the ensemble [13,14]. This motivated us to remove self-overlapping bubbles from the LCDT ensemble in $1+1$ dimensions too, and to check whether it makes a difference to the measurement of the Hausdorff dimension.

Detecting whether a self-overlapping bubble is created during the Monte Carlo simulation (and discarding the corresponding move) is nontrivial, since the property is nonlocal and requires a computationally expensive walk around the lattice (see [23] for details on implementation). For this reason we performed the numerical analysis on slightly smaller lattices of volume $N_{2} \leq 60.000$. Measurement of the average linear extension $\bar{r}$ in this setting, at $N_{2}=50.000$, still revealed a peak structure similar to that of standard LCDT with self-overlapping bubbles, providing evidence that this structure is not responsible for the observed instability. Proceeding like before to determine the Hausdorff dimension for this system, via finite-size scaling to maximize the overlap in shape (see Fig. 19) yielded a Hausdorff dimension of $d_{h}=3.10 \pm 0.2$.

To summarize, we have pinpointed an instability of the system with regard to its global behavior, due to occasional excursions to a globally elongated state, which can be observed by monitoring the geometry's average linear extension $\bar{r}$. This is the likely source of the suboptimal data quality for the measurement of the Hausdorff

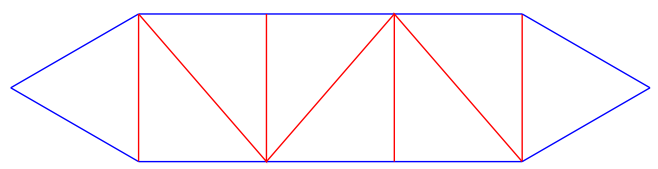

FIG. 17 (color online). A bubble has a sst-triangle at either end and arbitrarily many stt-triangles in between.

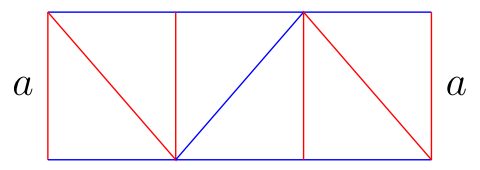

FIG. 18 (color online). A self-overlapping bubble; the links with label $a$ are to be identified. 


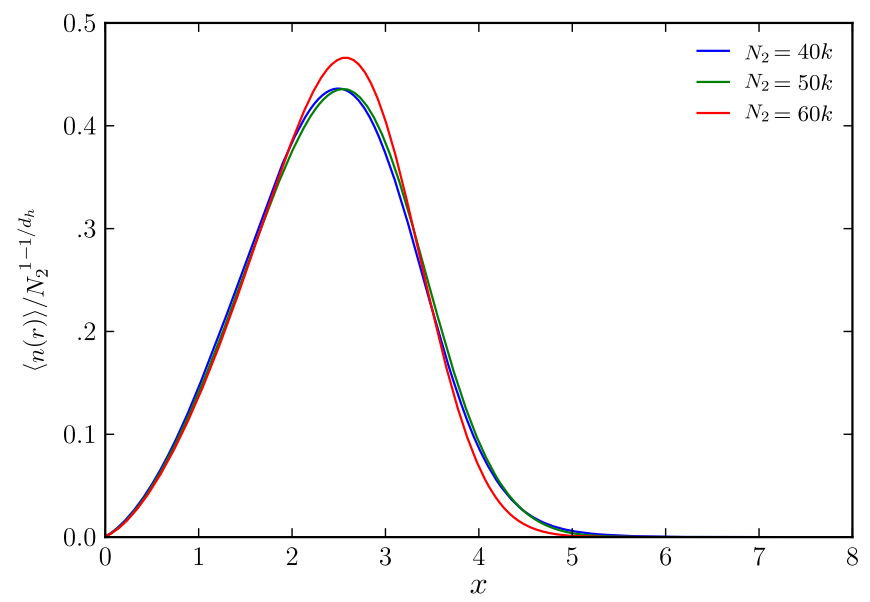

FIG. 19 (color online). LCDT quantum gravity without selfoverlapping bubbles: Fit for the best overlap of the rescaled shapes $\langle n(r)\rangle / N_{2}^{-1+1 / d_{h}}$ as a function of the rescaled distance $x=r / N_{2}^{1 / d_{h}}$, for Hausdorff dimension $d_{h}=3.1$.

dimension $d_{h}$. By considering a more general fitting function for extracting $d_{h}$ and by using a modified ensemble without self-overlapping bubbles we have found no hints of additional sources of error or a shift of the Hausdorff dimension toward the CDT value of 2 .

\section{CONCLUSIONS AND OUTLOOK}

The aim of our work was to measure observables in locally causal dynamical triangulations in two dimensions, most importantly, the spectral and the Hausdorff dimensions, and thereby understand the relation of LCDT to other models of two-dimensional quantum gravity based on dynamical triangulations. Our initial hypothesis was that LCDT lies in the same universality class as CDT, where both spectral and Hausdorff dimensions are equal to 2. While our measurement of LCDT's spectral dimension did yield a value compatible with 2 , with only a small error margin, this was not true for the Hausdorff dimension. Although the error bars were significantly larger - due to an instability in the system that persisted even at the largest volumes-our measurements found a Hausdorff dimension of $d_{h}=2.7 \pm$ 0.2 and $d_{h}=3.1 \pm 0.2$ for two slightly different variants of LCDT. On the basis of our simulations, it appears that LCDT is not equivalent to either DT or CDT in the continuum limit.

This would be an interesting result, because it implies the existence of a new universality class of two-dimensional quantum gravity in between Euclidean DT and Lorentzian CDT in two dimensions. The "in between" could be true quite literally, since within our measuring accuracy the Hausdorff dimension of LCDT is compatible with 3. A Hausdorff dimension $d_{h}=3$ has been observed previously, in simulations of CDT quantum gravity in $1+1$ dimensions coupled to eight copies of Ising spins [32] and coupled to several massless scalar fields [34], adding some plausibility to the possibility that a universality class with this property may actually exist. Further confirmation of the appearance of this new phase would come from locating a phase transition between CDT (corresponding to $\alpha=1 / 4$, at least for fixed volume) and LCDT as a function of the parameter $\alpha$ in our model. Having already invested considerable computing resources into the isotropic case $\alpha=1$ in the present work, we leave this investigation to a future publication. Needless to say, it would be extremely interesting to find an analytic solution of the LCDT model, to put our findings on a more definite footing.

\section{ACKNOWLEDGMENTS}

We thank J. Ambjørn for discussion. The contribution of R.L. is part of the research programme of the Foundation for Fundamental Research on Matter (FOM), financially supported by the Netherlands Organization for Scientific Research (NWO).

\section{APPENDIX GEOMETRIC SHAPE OF CONFIGURATIONS}

In this appendix, we give some more details about the instability we have observed in the LCDT system, which affects at least one observable, the average linear extension $\bar{r}$ of the Universe defined in (13), and contributes to the rather poor overlaps we have found in our finite-size scaling analysis to determine the Hausdorff dimension. Unlike other observables, which typically converge after about 300 sweeps, $\bar{r}$ does not, even for very large system size $N_{2}=400.000$ and after several thousands of sweeps. To understand better what happens geometrically, we have plotted the shape $n(r)$ of a typical configuration along the metastable bottom of the Monte Carlo history of $\bar{r}$ shown in Fig. 16, and of a configuration at one of the peaks. As illustrated by Fig. 20, the two are very different. Outside the peaks in $\bar{r}$, the shape of a configuration starts out with an almost linear increase until it reaches a single maximum, and then quickly drops to zero. A configuration from a peak
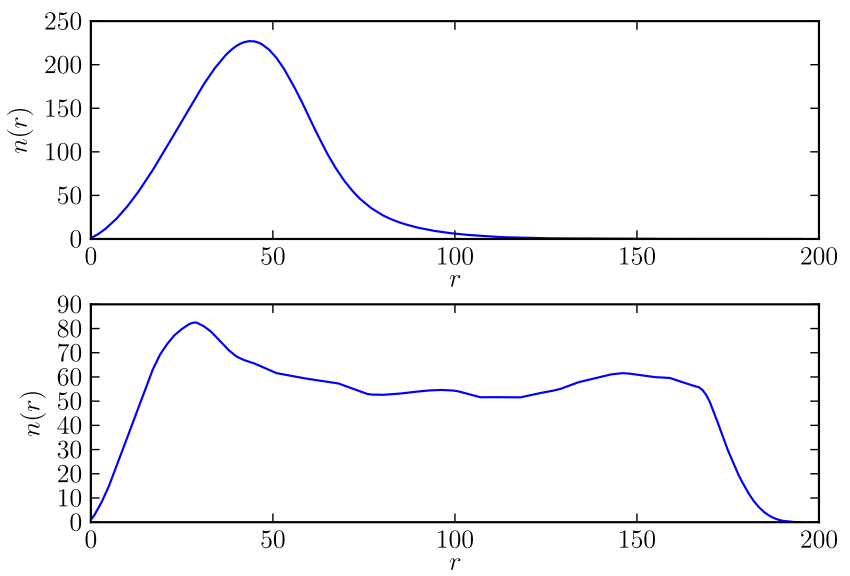

FIG. 20 (color online). Average shape $n(r)$ (top) and shape at peaks in $\bar{r}$ (bottom) at $N=9.000$, exhibiting a long plateau. 

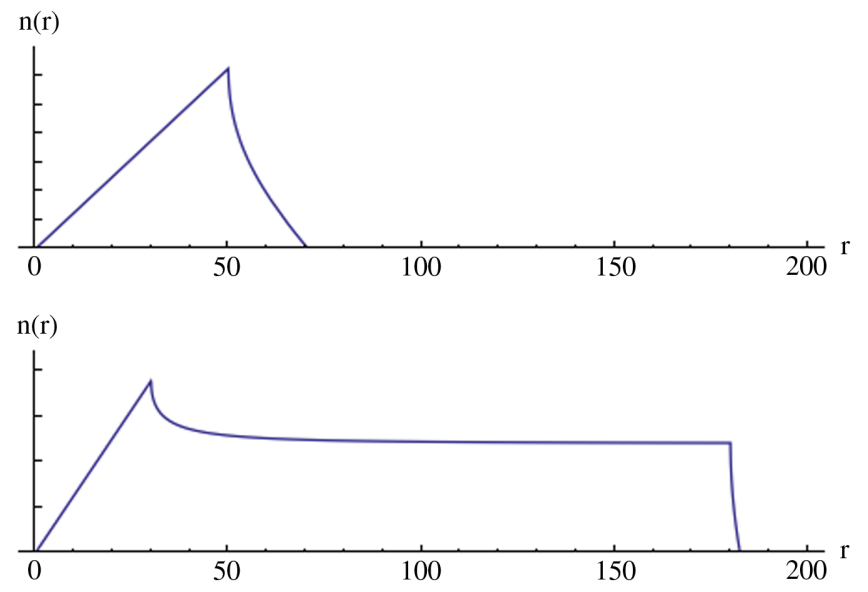

FIG. 21 (color online). Shape of a flat, classical torus in the continuum: square shaped of length 50 in either direction (top) and elongated with extension 30 and 180 in the two directions (bottom). in $\bar{r}$ also increases linearly until a first maximum, but then enters a long plateau before also going to zero. These two different shape functions are characteristic for a torus which is approximately square shaped (i.e. of a similar extension in either of the torus directions) and one which is elongated. This fact is illustrated by comparing the measured shapes with those of regular, flat tori ${ }^{14}$ in the continuum (Fig. 21). Despite the totally different setup (single, classical torus without local curvature and without quantum fluctuations), there is a clear qualitative resemblance with the shapes extracted from the full quantum simulation. Note that we have not attempted a proper translation between discrete and continuum units of length and volume, which would be necessary for a quantitative comparison.

\footnotetext{
${ }^{14}$ For simplicity, we are considering only tori which are obtained from gluing flat rectangles without any "twists."
}

[1] J. Ambjørn, A. Görlich, J. Jurkiewicz, and R. Loll, Nonperturbative quantum gravity, Phys. Rep. 519, 127 (2012).

[2] S. Catterall, G. Thorleifsson, J. B. Kogut, and R. Renken, Singular vertices and the triangulation space of the $\mathrm{D}$ sphere, Nucl. Phys. B468, 263 (1996); P. Bialas, Z. Burda, B. Petersson, and J. Tabaczek, Appearance of mother universe and singular vertices in random geometries, Nucl. Phys. B495, 463 (1997).

[3] P. Bialas, Z. Burda, A. Krzywicki, and B. Petersson, Focusing on the fixed point of four-dimensional simplicial gravity, Nucl. Phys. B472, 293 (1996); B. V. de Bakker, Further evidence that the transition of four-dimensional dynamical triangulation is first order, Phys. Lett. B 389, 238 (1996).

[4] J. Ambjørn, L. Glaser, A. Görlich, and J. Jurkiewicz, Euclidian four-dimensional quantum gravity with a nontrivial measure term, J. High Energy Phys. 10 (2013) 100; D. Coumbe and J. Laiho, Exploring Euclidean dynamical triangulations with a nontrivial measure term, J. High Energy Phys. 04 (2015), 028; T. Rindlisbacher and P. de Forcrand, Euclidean dynamical triangulation revisited: Is the phase transition really 1st order? J. High Energy Phys. 05 (2015), 138.

[5] J. Ambjørn, A. Görlich, J. Jurkiewicz, and R. Loll, Planckian Birth of the Quantum de Sitter Universe, Phys. Rev. Lett. 100, 091304 (2008); The nonperturbative quantum de Sitter universe, Phys. Rev. D 78, 063544 (2008).

[6] J. Ambjørn, S. Jordan, J. Jurkiewicz, and R. Loll, A SecondOrder Phase Transition in CDT, Phys. Rev. Lett. 107, 211303 (2011); Second- and first-order phase transitions in CDT, Phys. Rev. D 85, 124044 (2012).
[7] J. Ambjørn, A. Görlich, J. Jurkiewicz, A. Kreienbuehl, and R. Loll, Renormalization group flow in CDT, Classical Quantum Gravity 31, 165003 (2014).

[8] J. Ambjørn and R. Loll, Nonperturbative Lorentzian quantum gravity, causality and topology change, Nucl. Phys. B536, 407 (1998).

[9] F. David, in Gravitation and Quantizations, edited by B. Julia and J. Zinn-Justin (North-Holland, Amsterdam, 1995), p. 679; J. Ambjørn, B. Durhuus, and T. Jonsson, Quantum Geometry. A Statistical Field Theory Approach (Cambridge University Press, Cambridge, 1997).

[10] J. Ambjørn, R. Loll, J. L. Nielsen, and J. Rolf, Euclidean and Lorentzian quantum gravity: Lessons from two dimensions, Chaos Solitons Fractals 10, 177 (1999).

[11] P. H. Ginsparg and G. W. Moore, in Recent Directions in Particle Theory, edited by J. Harvey and J. G. Polchinski (World Scientific, Singapore, 1993), p. 277.

[12] J. Ambjørn, L. Glaser, Y. Sato, and Y. Watabiki, Twodimensional CDT is two-dimensional Hořava-Lifshitz quantum gravity, Phys. Lett. B 722, 172 (2013).

[13] S. Jordan, Ph.D. thesis, Radboud University, 2013.

[14] S. Jordan and R. Loll, Causal dynamical triangulations without preferred foliation, Phys. Lett. B 724, 155 (2013); De Sitter universe from causal dynamical triangulations without preferred foliation, Phys. Rev. D 88, 044055 (2013).

[15] P. Di Francesco, E. Guitter, and C. Kristjansen, Integrable two-dimensional Lorentzian gravity and random walks, Nucl. Phys. B567, 515 (2000).

[16] P. Di Francesco, E. Guitter, and C. Kristjansen, Generalized Lorentzian gravity in $(1+1)$ dimensions and the Calogero Hamiltonian, Nucl. Phys. B608, 485 (2001). 
[17] J. Ambjørn and A. Ipsen, Universality of two-dimensional causal dynamical triangulations, Phys. Lett. B 724, 150 (2013).

[18] J. Ambjørn, R. Loll, W. Westra, and S. Zohren, Putting a cap on causality violations in CDT, J. High Energy Phys. 12 (2007) 017; J. Ambjørn, R. Loll, Y. Watabiki, W. Westra, and S. Zohren, A String field theory based on causal dynamical triangulations, J. High Energy Phys. 05 (2008) 032 .

[19] J. Ambjørn and T. G. Budd, Trees and spatial topology change in CDT, J. Phys. A 46, 315201 (2013).

[20] B. Durhuus and C. W. H. Lee, A string bit Hamiltonian approach to two-dimensional quantum gravity, Nucl. Phys. B623, 201 (2002).

[21] J. Ambjørn, J. Jurkiewicz, and R. Loll, Dynamically triangulating Lorentzian quantum gravity, Nucl. Phys. B610, 347 (2001).

[22] R. Hoekzema, Master thesis, University of Utrecht, 2012.

[23] B. Ruijl, Masters thesis, Radboud University Nijmegen, 2013, http://www.ru.nl/publish/pages/760962/thesis_final _version.pdf.

[24] J. Ambjørn, J. Correia, C. Kristjansen, and R. Loll, On the relation between Euclidean and Lorentzian two-dimensional quantum gravity, Phys. Lett. B 475, 24 (2000).

[25] J. Ambjørn, K. N. Anagnostopoulos, and R. Loll, A new perspective on matter coupling in two-dimensional quantum gravity, Phys. Rev. D 60, 104035 (1999).
[26] H. Kawai, N. Kawamoto, T. Mogami, and Y. Watabiki, Transfer matrix formalism for two-dimensional quantum gravity and fractal structures of spacetime, Phys. Lett. B 306, 19 (1993); J. Ambjørn and Y. Watabiki, Scaling in quantum gravity, Nucl. Phys. B445, 129 (1995).

[27] J. Ambjørn, J. Jurkiewicz, and Y. Watabiki, On the fractal structure of two-dimensional quantum gravity, Nucl. Phys. B454, 313 (1995).

[28] B. Durhuus, T. Jonsson, and J. F. Wheater, On the spectral dimension of causal triangulations, J. Stat. Phys. 139, 859 (2010).

[29] J. Ambjørn, J. Jurkiewicz, and R. Loll, Reconstructing the Universe, Phys. Rev. D 72, 064014 (2005).

[30] J. Ambjørn, J. Jurkiewicz, and R. Loll, Spectral Dimension of the Universe, Phys. Rev. Lett. 95, 171301 (2005).

[31] D. Benedetti and J. Henson, Spectral geometry as a probe of quantum spacetime, Phys. Rev. D 80, 124036 (2009).

[32] J. Ambjørn, K. N. Anagnostopoulos, and R. Loll, Crossing the $\mathrm{c}=1$ barrier in two-dimensional Lorentzian quantum gravity, Phys. Rev. D 61, 044010 (2000).

[33] S. Catterall, G. Thorleifsson, M. J. Bowick, and V. John, Scaling and the fractal geometry of two-dimensional quantum gravity, Phys. Lett. B 354, 58 (1995).

[34] J. Ambjørn, A. T. Görlich, J. Jurkiewicz, and H. G. Zhang, Pseudotopological transitions in two-dimensional gravity models coupled to massless scalar fields, Nucl. Phys. B863, 421 (2012). 